\title{
Cytomegalovirus infection is not a major cause of corneal graft failure in the United Kingdom
}

\author{
Claudia A. da Costa Paula ${ }^{1} \cdot$ Daniel M. Gore ${ }^{1} \cdot K_{\text {Khilan Shah }}{ }^{2} \cdot$ Geert Kuit $^{2} \cdot$ Romesh I. Angunawela $^{1} \cdot$ \\ James P. Barnett ${ }^{3}$. Stephen J. Tuft ${ }^{1}$
}

Received: 10 May 2018 / Revised: 4 December 2018 / Accepted: 13 December 2018 / Published online: 8 January 2019

(c) The Royal College of Ophthalmologists 2019

\begin{abstract}
Background Cytomegalovirus (CMV) endotheliitis is a significant cause for acute corneal allograft rejection in East Asian populations, where there is a high CMV seroprevalence. To determine how frequently CMV is associated with corneal graft failure in the UK, we looked for the presence of CMV DNA in grafts that had failed and had been removed at repeat keratoplasty. We also looked for CMV DNA in corneal rims discarded after corneal transplantation.

Methods In this retrospective study, we identified 54 cases of corneal graft failure following endothelial rejection and 38 control grafts that had failed without a history of endothelial rejection. For these groups archived formalin-fixed paraffin-embedded (FFPE) tissue samples were retrieved. We also prospectively examined 80 non-fixed cornea rims following transplantation surgery. In all samples nested quantitative PCR was used to identify CMV, herpes simplex virus (HSV) and varicella zoster virus (VZV) DNA. We also used in situ hybridisation to examine for CMV DNA in the FFPE samples.

Results No CMV or VZV DNA was detected in any of the archived case or control FFPE tissues. One corneal rim from the control group was positive for HSV. In situ hybridisation for CMV was negative for CMV in all FFPE samples. No CMV, VZV or HSV DNA was detected in the donor corneal rim samples.

Conclusion CMV DNA was not identified in excised failed corneal tissue or from tissue prior to transplantation. We infer that CMV infection is not a significant factor risk for corneal graft failure in the United Kingdom.
\end{abstract}

\section{Introduction}

Cytomegalovirus (CMV), or human herpes virus type 5, is a common human pathogen with a high seroprevalence in the United Kingdom (UK) population, ranging from $49 \%$ in White women born in the UK to 98\% in South Asian women born in Asia [1]. CMV rarely causes clinical disease in immunocompetent individuals, although active CMV infection is common in solid-organ transplant recipients, especially if there is a HLA-DR mismatch or lympholytic immunosuppression [2]. The majority of reports of CMV infection in corneal transplant recipients (CMV endotheliitis) are from

Daniel M. Gore

daniel.gore1@nhs.net

1 External Disease Service, Moorfields Eye Hospital, London, United Kingdom

2 Eye Bank, Moorfields Eye Hospital, London, United Kingdom

3 On behalf of the Micropathology Ltd Research Group, University of Warwick Science Park, Coventry, United Kingdom
East Asia [3-10]. The clinical signs include corneal oedema and keratic precipitates with or without anterior chamber (AC) inflammation, which can mimic acute allograft rejection. Identifying an acute infective cause when there is a suspected acute graft rejection is crucial, because intensive topical corticosteroid treatment (as used for rejection) may exacerbate CMV endotheliitis, increasing the risk of eventual graft failure.

Corneal allograft rejection remains a significant problem, occurring in $20 \%$ of eyes at 5 years [11]. The reports from South Asia that CMV infection can mimic acute allograft rejection prompted us to investigate whether there was evidence in the UK of CMV infection in corneal tissue following graft failure.

\section{Methods}

\section{Archived samples}

To investigate the historical prevalence of CMV infection in failed corneal grafts we used the search terms 'rejection' or 
'rejected' to search a national ophthalmic pathology referral service database for samples logged between 1998 and 2014. Pathology reports were screened by two investigators (DMG and CdCP) to confirm inclusion. We also reviewed the medical records to extract clinical data and confirm the presence of a previous endothelial rejection event. We included failed penetrating and Descemet's stripping automated endothelial keratoplasty buttons (DSAEK), but excluded anterior lamellar grafts and eyes with concomitant microbial keratitis or endophthalmitis. Since the introduction of endothelial keratoplasty at our institution for reviving failed penetrating grafts, the DSAEK button (or more recently DMEK) has been applied directly on to the PK endothelium. In these eyes, tissue is therefore not available for histological analysis. Failed grafts without a documented history of endothelial rejection served as controls. Formalin-fixed paraffin-embedded (FFPE) tissue samples were then retrieved for testing.

\section{Fresh samples}

To estimate the prevalence of CMV DNA in corneal graft tissue we prospectively tested 80 fresh tissues processed for corneal transplantation by Moorfields Eye Hospital eye bank. Whole corneal rims returned to the eye bank after completion of surgery were placed in a viral transport medium (Virocult ${ }^{\circledR}$, Medical Wire \& Equipment, Corsham, UK).

\section{Polymerase chain reaction}

FFPE samples (both cases and controls) were chemically deparaffinised by hand, using a series of Xylene, ethanol and water washes. The samples were then subjected to a rigorous protease digestion before proceeding with DNA extraction on the Qiasymphony biorobot. First round amplification by polymerase chain reaction (PCR) of herpes simplex virus (HSV), varicella zoster virus (VZV) and CMV nucleic acids was performed using a volume of $50 \mu \mathrm{L}$ reaction with $30 \mu \mathrm{L}$ PCR master mix and $20 \mu \mathrm{L}$ nucleic acid extraction on a block thermocycler. Second round PCR was carried out using $24 \mu \mathrm{L}$ PCR master mix and $1 \mu \mathrm{L}$ of first round product (Roche Lightcycler 480), with continuing acquisition of fluorescence signal during PCR and melt curve analysis. HSV, VZV and CMV PCR uses in-house PCR master mixes containing Supertherm taq-polymerase, dNTP's and PCR buffer in the first round, with addition of EvaGreen fluorescent dye in the second round. We used the following primers: glycoprotein B gene $g B$ (CMV), ssDNAbinding protein gene found on ORF29 (VZV) and glycoprotein D gene (HSV I \& II). Positive control nucleic acid for both HSV and VZV was extracted from cell culture isolates of wild-type virus. Positive control CMV nucleic acid were tenfold dilutions extracted from proteinase $\mathrm{K}$ digested infected MRC-5 cell lines ranging from $10^{3}$ to $10^{6}$ genome copies $/ \mathrm{mL}$. We validated the nucleic extraction process from FFPE tissue using an archived retinal sample from an eye enucleated for CMV-retinitis. PhiX PCR inhibition assays were additionally performed for all samples indicating that there was no inhibition of PCR.

\section{In situ hybridisation (ISH)}

Three tissue sections ( $4 \mu \mathrm{m}$ thick) from each FFPE block were placed onto Leica Bond Plus adhesive slides, baked for $30 \mathrm{~min}$ at $60^{\circ} \mathrm{C}$, cooled, dewaxed, rehydrated and enzymatically digested (Bond enzyme $1,15 \mathrm{~min}$ at $37^{\circ} \mathrm{C}$ ) prior to in situ staining. CMV positivity was visualised using a standard ISH Protocol (Protocol A) on a Leica BondMax automated immune-stainer, with the following core reagents: CMV Probe (Leica PB0614), CMV negative (Leica PB0809), anti-fluorescein (Leica AR0833) and Bond Polymer Refine Red Detection (Leica DS9390). Dehydrated slides were mounted on a Leica CV500 coverslip with Pertex. Funding was not available for ISH testing on fresh corneal rim samples.

\section{Clinical interpretation}

A CMV-positive result was defined as clinically significant if the same tissue sample was also negative for HSV and VZV.

The work was conducted with ethical approval from The Moorfields Biobank Internal Ethics Committee (10/H0106/ 57-2014ETR42).

\section{Results}

\section{Archived samples}

A search of the pathology database identified 263 tissue sample reports that contained either 'rejection' or 'rejected.' All reports were manually screened for further inclusion from which 25 were excluded as not relevant (corneal biopsy, impression cytology, vitreous, evisceration). Of the remaining 238, 132 were from 116 patients were treated at Moorfields Eye Hospital and thus had available clinical records. The medical records of 104 $(90 \%)$ patients could be traced. We then confirmed a clinical history of rejection in 54 samples of 48 patients: 5 patients had two grafts that failed following rejection and 1 patient had 3 grafts that had failed. There were 41 grafts of 38 patients that had failed without a documented rejection episode ( 3 patients had 2 failed grafts). A total of 18 patients were excluded, because they did not meet the inclusion criteria or the clinical notes lacked sufficient 
Fig. 1 Accountability flow diagram for archived formalinfixed paraffin-embedded tissue samples

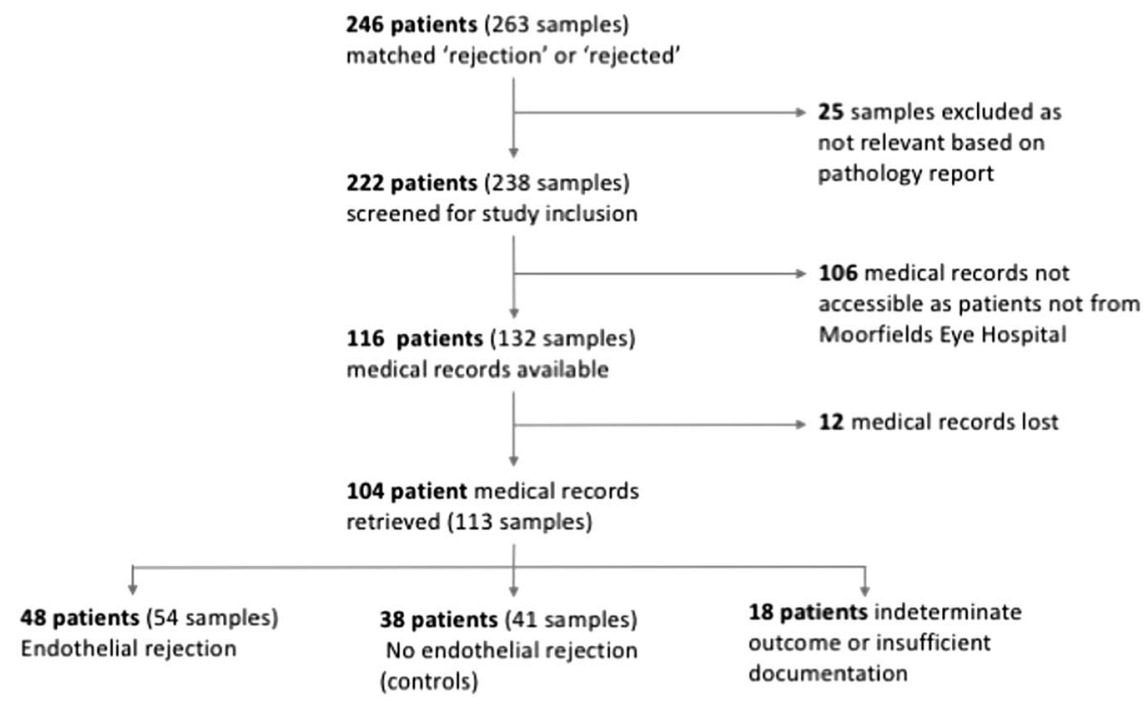

Table 1 Self-reported patient ethnicity for 86 pathological specimens examined for PCR and ISH

\begin{tabular}{lcc}
\hline Ethnicity & Control & Rejection \\
\hline British/White other & 21 & 24 \\
Black African/Caribbean & 2 & 5 \\
Indian/Pakistani & 5 & 6 \\
Chinese/Asian other & 2 & 1 \\
Mixed background & 1 & 1 \\
Not stated & 7 & 11 \\
Total & 38 & 48 \\
\hline
\end{tabular}

details to confirm or exclude a rejection episode (Fig. 1, Table 1).

CMV DNA was detected in the sections from the individual with a known history of CMV retinitis (positive control). However, no CMV or VZV DNA was detected in any sample from either the rejection or control groups. One control sample was positive for HSV. ISH confirmed that all samples were negative for CMV.

\section{Fresh samples}

We examined 80 fresh corneal rims. These were collected between September and November 2015, and March and July 2017. No CMV, VZV or HSV DNA was detected in any of the donor corneal rims.

\section{Discussion}

CMV endotheliitis has been reported as a cause for corneal graft failure, predominantly in case series from East Asia [3-10]. CMV endotheliitis is very uncommon in the UK in the absence of severe systemic immunosuppression (HIV AIDS). One report exists in the literature of a BritishChinese patient who developed severe CMV-positive endotheliitis with uveitis post-phacoemulsification [12]. One author (SJT) has seen CMV endotheliitis in non-grafted immunocompetent patients only twice in more than 25 years of clinical practice in the UK. The clinical picture of endotheliitis may be characteristic, with focal endothelial precipitates and corneal oedema, but it may mimic an acute allograft rejection and lead to graft failure. Perversely, topical corticosteroid, which is the mainstay of treatment for allograft rejection, may make the endotheliitis worse if it is not accompanied by effective antiviral cover. Cases may also be misdiagnosed as HSV or VZV keratitis, when the usual therapy for herpes virus infection may be ineffective. The majority of cases are probably the result of reactivation of latent CMV infection in the host precipitated by surgery or topical steroid, although CMV may also be transmitted by donor material [13]. In this paper we used PCR and ISH to investigate whether CMV can be detected in corneal tissue after graft failure and in tissue cleared for corneal transplantation. We found no evidence of CMV in any of the 166 samples tested.

Testing for CMV infection in cases where there is corneal endothelial graft rejection is not routine practice in the UK. This is in part because the test is performed on aqueous fluid, and an anterior chamber paracentesis is an invasive procedure, but also because accurate PCRbased tests for CMV have historically not been readily available. On the basis of our study, we do not recommend a change in practice unless there are atypical signs on examination. This may also apply to other countries with a similar CMV seroprevalence to the UK. While prior exposure to CMV differs between the UK and South Asia (49\% vs. 98\% seroprevalence), 
immunological evidence in a peripheral blood sample doesn't explain why intraocular CMV infection rates (detected by DNA) differ in the two populations. Cases with an atypical presentation, known prior seropositivity to $\mathrm{CMV}$, cases that do not respond to corticosteroid, or cases in which there has been recurrent early corneal graft failure may warrant further investigation $[10,14,15]$. Similarly, testing for CMV infection in corneal donor tissue, or CMV seropositivity in corneal donors, is not performed by UK eye banks as CMV is not considered to be a common pathogen transferred by corneal transplantation. On the basis of our study, in which no viral DNA was detected in 80 fresh donor corneas, we do not recommend a change in practice.

In conclusion, our results suggest that CMV endotheliitis is not a significant cause of corneal graft failure in the UK. However, there are a number of methodological limitations to this study. Although FFPE tissue is an excellent source of DNA, its extraction remains a challenge. Nucleic acids are cross-linked and fragmented by formaldehyde during the fixation process, which can make it more difficult to amplify DNA. Despite this, assays on all samples indicated that there was no inhibition of amplification during PCR. DNA fragmentation can also occur during fixation, which can be problematic when using relatively large primers such as those used for the glycoprotein B gene. To exclude this as a cause for failure to detect CMV DNA we performed a positive control using a FFPE tissue sample from a patient with confirmed CMV-retinitis. In this sample the presence of CMV nucleic acid was confirmed. Nonetheless, a more robust approach would be to test freshly excised failed graft tissue, transported in viral culture media, the results of which would help define whether a change to current clinical practice is warranted or not.

Finally, it is also possible that CMV DNA may not persist in corneal tissue and may not be detectable if there is an interval between infection and repeat graft surgery.

\section{Summary}

\section{What was known before}

- CMV endotheliitis is an established cause of acute corneal graft rejection in East Asian populations. CMVmediated graft rejection is difficult to treat and often results in eventual graft failure.

\section{What this study adds}

- CMV DNA was not detected in any excised failed corneal grafts from the United Kingdom. This suggests
CMV infection is not a significant risk factor in graft rejection and failure in this population

Acknowledgements The authors wish to thank Lucretia Medard of the Institute of Ophthalmology Pathology Department for help searching the archives and retrieving specimens, and David Essex of the same department for preparation of ISH slides. D.M.G. and S.J.T. acknowledge that a proportion of their financial support is from the NIHR Biomedical Research Centre at Moorfields Eye Hospital NHS Foundation Trust and UCL Institute of Ophthalmology and the NIHR Moorfields Clinical Research Facility. Additional funding received from Fight for Sight (1550/51); Sir Halley Steward Trust. The views expressed are those of the authors and not necessarily those of the NHS, the NIHR or the Department of Health.

\section{Compliance with ethical standards}

Conflict of interest The authors declare that they have no conflict of interest.

Publisher's note: Springer Nature remains neutral with regard to jurisdictional claims in published maps and institutional affiliations.

\section{References}

1. Pembrey L, Raynor P, Griffiths P, Chaytor S, Wright J, Hall AJ. Seroprevalence of cytomegalovirus, epstein barr virus and varicella zoster virus among pregnant women in Bradford: A Cohort Study. PLoS ONE. 2013;8:e81881.

2. van der Bij W, Speich R. Management of cytomegalovirus infection and disease after solid-organ transplantation. Clin Infect Dis. 2001;33(Suppl 1):S32-7.

3. Chu HY, Sun CC, Chuang WY, Liou SW, Ma DHK, Lai CC, et al. Cytomegalovirus associated corneal endotheliitis after penetrating keratoplasty in a patient with Fuchs corneal endothelial dystrophy. Br J Ophthalmol. 2012;96:300-1.

4. Anshu A, Chee S-P, Mehta JS, Tan DTH. Cytomegalovirus endotheliitis in descemet's stripping endothelial keratoplasty. Ophthalmology. 2009;116:624-30.

5. Suzuki T, Hara Y, Uno T, Ohashi Y. DNA of cytomegalovirus detected by PCR in aqueous of patient with corneal endotheliitis after penetrating keratoplasty. Cornea. 2007;26:370-2.

6. Wang S-C, Tsai I-L, Lin H-C, Kuo L-L, Tsai C-Y, Liou S-W. Recurrent cytomegalovirus corneal endotheliitis after penetrating keratoplasty. Eur J Ophthalmol. 2010;20:457-9.

7. Chee S-P, Jap A, Ling ECW, Ti S-E. Cytomegalovirus-positive corneal stromal edema with keratic precipitates after penetrating keratoplasty: a case-control study. Cornea. 2013;32:1094-8.

8. Kandori M, Inoue T, Takamatsu F, Kojima Y, Hori Y, Maeda N, et al. Prevalence and features of keratitis with quantitative polymerase chain reaction positive for cytomegalovirus. Ophthalmology. 2010;117:216-22.

9. Sonoyama H, Araki-Sasaki K, Osakabe Y, Nakamura M, Amano $\mathrm{S}$, Koizumi N, et al. Detection of cytomegalovirus DNA from cytomegalovirus corneal endotheliitis after penetrating keratoplasty. Cornea. 2010;29:683-5.

10. Shahrudin NA, Mohd Zahidin AZ, Md Noh UK, Wan Abdul Halim WH, Md Din N. CMV endotheliitis: a cause for recurrent failed corneal transplant. GMS Ophthalmol Cases. 2017;7:Doc31.

11. Tan DDT, Dart JK, Holland EJ, Kinoshita S. Corneal transplantation. Lancet. 2012;379:1749-61. 
12. Ghauri A-J, Williams GP, Shah S, Murray PI, Rauz S. Post-phacoemulsification cytomegalovirus corneal endotheliitis diagnosis and management. JRSM Short Rep. 2012; $3: 1-4$.

13. Holland EJ, Bennett SR, Brannian R, Osborne JC, Goeken JA, Krachmer JH. The risk of cytomegalovirus transmission by penetrating keratoplasty. Am J Ophthalmol. 2018;105: $357-60$.
14. Koizumi N, Suzuki T, Uno T, Chihara H, Shiraishi A, Hara Y, et al. Cytomegalovirus as an etiologic factor in corneal endotheliitis. Ophthalmology. 2008;115:292-e3.

15. Koizumi N, Inatomi T, Suzuki T, Shiraishi A, Ohashi Y, Kandori $\mathrm{M}$, et al. Clinical features and management of cytomegalovirus corneal endotheliitis: analysis of 106 cases from the Japan corneal endotheliitis study. Br J Ophthalmol. 2014. Available at: http:// bjo.bmj.com/cgi/doi/10.1136/bjophthalmol-2013-304625 